\section{Aplicación de la rúbrica como instrumento de evaluación de la competencia clínica de broncoscopia del residente de cirugía torácica}

\section{Application of the rubric as an instrument for assessing the clinical competence of bronchoscopy of the thoracic surgery resident}

\author{
Juan Carlos Vázquez Minero,* Telésforo Medina Otero, ${ }^{\ddagger}$ Arturo Chávez Tinoco,* \\ Marco Antonio Iñiguez García, José Morales Gómez*
}

*Instituto Nacional de Enfermedades Respiratorias Ismael Cosío Villegas, Ciudad de México, México;

`Universidad Simón Bolívar, México.

\begin{abstract}
RESUMEN. Dentro de las competencias profesionales de los residentes de cirugía torácica se encuentra la realización de broncoscopias, pero para valorar éstas es necesario un adecuado instrumento, que en este caso es la rúbrica. Material y métodos: Se seleccionaron a los residentes de cirugía de tórax, cuatro de segundo año y cuatro de cuarto año para aplicarles la rúbrica, y a un cirujano de cinco años de experiencia y uno de 40 años de experiencia como evaluadores. Se aplicó la rúbrica en una sola ocasión, evaluando un cirujano a cada residente. Resultados: Se observó al aplicar la rúbrica que todos los residentes cayeron en el rango de competentes, pero los residentes de segundo año aún no completaban al 100\% los puntos que la rúbrica evalúa y los de cuarto año sí eran al 100\% competentes. Conclusión: La rúbrica fue un instrumento reproducible y útil para evaluar de manera fidedigna al residente, se observó mejor dominio de la competencia clínica en los residentes de tercer año a comparación de los de primer año.
\end{abstract}

Palabras clave: Competencia, rúbrica, evaluación.

\section{INTRODUCCIÓN}

La actividad profesional de un cirujano de tórax en nuestro país exige el amplio dominio de conceptos teóricos, habilidades técnicas y actitudes, es decir, de lo que conocemos como competencias profesionales, en donde una de ellas es la realización de broncoscopias, tanto para diagnóstico como para tratamiento en las pa-

Correspondencia:

Dr. Juan Carlos Vázquez Minero

Instituto Nacional de Enfermedades Respiratorias

Ismael Cosío Villegas, Ciudad de México.

Correo electrónico: minerojc@hotmail.com

Trabajo recibido: 05-VIII-2019; aceptado: 20-III-2020.
ABSTRACT. Residents of thoracic surgery within their professional skills are performing bronchoscopies, but to assess these is necessary an appropriate instrument that in this case is the rubric. Material and methods: Residents of four second and four quarter year chest surgery were selected to apply the rubric, and a surgeon of 5 years of experience and one of 40 years of experience as evaluators. The rubric was applied on only one occasion, evaluating each resident a surgeon. Results: It was observed when applying the rubric that all residents fell into the competent range, but second year residents still did not complete $100 \%$ the points that the rubric evaluates and those of quarter year if they were $100 \%$ competent. Conclusion: The rubric was a reproducible and useful instrument, to reliably assess the resident and where better dominance of the clinical competence was observed in the third year residents compared to the first year residents.

Keywords: Competence, rubric, evaluation.

tologías respiratorias que sean susceptibles de atenderse mediante esta técnica.

La Facultad de Medicina de la Universidad Nacional Autónoma de México (UNAM) define como competencia lo siguiente: conjunto de conocimientos, habilidades, actitudes, valores y aptitudes que interrelacionados entre sí permiten tener un desempeño profesional eficiente de conformidad con el estado del arte.

Desde el punto de vista de la cirugía, las competencias que debe tener un profesional quirúrgico son: pericia técnica, profesionalismo, abogacía en salud, comunicación, trabajo en equipo, gerencia y liderazgo, erudición, enseñanza, juicio y toma de decisiones. Algunas de estas competencias pueden ser evaluadas con una rúbrica, en particular, la pericia técnica, la toma de decisiones y el trabajo en equipo. ${ }^{2}$ 
Las competencias se van adquiriendo durante sus cuatro años de entrenamiento al realizar cotidianamente, acorde a su nivel de dominio, estudios broncoscópicos. En la mayoría de los casos, la evaluación de dichas actividades queda en el criterio personal del evaluador, es decir, del cirujano que entra con ellos al procedimiento, sin tener una guía que sea útil, clara, reproducible y, sobre todo, tenga validez para determinar el grado de competencia de esta actividad profesional.

La rúbrica es una evaluación auténtica centrada en el desempeño del alumno, o sea en las competencias del residente de cirugía de tórax y se define como: «Guías o escalas de evaluación en donde se establecen niveles progresivos de dominio o pericia relativos al desempeño que una persona muestra respecto de un proceso o producción determinada. Las rúbricas integran un amplio rango de criterios que cualifican de modo progresivo el tránsito de un desempeño incipiente de novato al grado de experto». ${ }^{3}$

Es importante destacar que parte de la evaluación en medicina, en particular de cirugía, es el grado de avance en el aprendizaje y es ahí donde la rúbrica juega un papel importante en este proceso ya que permite la evaluación in situ de la práctica y la observación y valoración de la competencia. ${ }^{4}$

En este artículo aplicamos la rúbrica para ver su utilidad en la evaluación de la competencia de broncoscopia en residentes de cirugía de tórax.

\section{MATERIAL Y MÉTODOS}

Se aplicó una rúbrica a cuatro residentes de segundo y cuarto año de la residencia de cirugía de tórax del Institu- to Nacional de Enfermedades Respiratorias (INER) Ismael Cosío Villegas durante la realización de una broncoscopia en pacientes reales por dos profesores del curso de cirugía de tórax del Instituto. La finalidad fue tener dos visiones del fenómeno a evaluar que es la competencia en broncoscopia de los residentes.

El profesor 1 tiene cinco años de experiencia como adscrito; el profesor 2 tiene 40 años de experiencia como cirujano de tórax y es uno de los fundadores de la escuela quirúrgica del instituto y formador de muchas generaciones de especialistas.

La finalidad de esta metodología es comparar lo observado por los dos profesores, con visiones y experiencias diferentes dentro de la docencia en cirugía de tórax y ver la utilidad de la rúbrica como instrumento de evaluación.

\section{RESULTADOS}

Después de un año de realizar broncoscopias por parte de los residentes de segundo año y de tres años por parte de los residentes de cuarto año se aplicó la rúbrica (Tabla 1). Ésta evaluaba seis aspectos, cada uno con una escala de valores de 5, 8 y 10 puntos. De tal manera que la sumatoria da una escala de evaluación de 30-39 puntos no competente; de 40-49 parcialmente competente; y de 50-60 puntos competente.

Para la aplicación de este instrumento, se obtuvieron los datos de dos profesores de la especialidad. El evaluador 1 , un profesor con cinco años de experiencia quirúrgica; el evaluador 2, un profesor con 40 años de experiencia.

Los resultados en general fueron competentes para todos los residentes, tanto de cuarto año como de segundo. Los

Tabla 1: Rúbrica para evaluación de competencias durante la realización de broncoscopia.

\begin{tabular}{|c|c|c|c|}
\hline Nivel & $\mathbf{1}$ & $\mathbf{2}$ & $\mathbf{3}$ \\
\hline $\begin{array}{c}\text { Preparación } \\
\text { del alumno }\end{array}$ & $\begin{array}{c}\text { No se lava las manos ni calza } \\
\text { guantes (5) }\end{array}$ & $\begin{array}{c}\text { Sólo se calza guantes y } \\
\text { cubrebocas (8) }\end{array}$ & $\begin{array}{c}\text { Se lava las manos y calza guantes, utiliza } \\
\text { cubrebocas, gorro y lentes de protección (10) }\end{array}$ \\
\hline $\begin{array}{c}\text { Preparación } \\
\text { del equipo }\end{array}$ & $\begin{array}{c}\text { No checa la esterilidad y limpieza } \\
\text { del broncoscopio (5) }\end{array}$ & $\begin{array}{c}\text { Tiene el broncoscopio limpio, pero } \\
\text { no checa esterilidad (8) }\end{array}$ & Tiene el broncoscopio limpio y estéril (10) \\
\hline $\begin{array}{c}\text { Preparación } \\
\text { del paciente }\end{array}$ & $\begin{array}{c}\text { El paciente solo está acostado en } \\
\text { la mesa quirúrgica (5) }\end{array}$ & $\begin{array}{c}\text { El paciente tiene colocada solo la } \\
\text { capelina (8) }\end{array}$ & $\begin{array}{c}\text { El paciente tiene colocada la capelina y la } \\
\text { boquilla o bien mascarilla laríngea lista (10) }\end{array}$ \\
\hline $\begin{array}{c}\text { Preparación } \\
\text { de la vía } \\
\text { aérea }\end{array}$ & $\begin{array}{c}\text { No aplica medicamento (5) } \\
\text { Revisión de } \\
\text { la vía aérea } \\
\text { superior }\end{array}$ & $\begin{array}{c}\text { No revisa e introduce broncoscopio (5) } \\
\text { a oximetazolina solamente }\end{array}$ & $\begin{array}{c}\text { Aplica lidocaína local cuando es por vía oral, } \\
\text { oximetazolina cuando es vía nasal y además } \\
\text { lidocaína por el broncoscopio (10) }\end{array}$ \\
\hline $\begin{array}{c}\text { Revisión de } \\
\text { la tráquea y } \\
\text { bronquios }\end{array}$ & $\begin{array}{c}\text { Revisa la tráquea y los bronquios } \\
\text { principales y segmentos bronquia- } \\
\text { les sin decir nomenclatura (5) }\end{array}$ & $\begin{array}{c}\text { Revisa la tráquea y los bronquios } \\
\text { principales y segmentos bronquiales } \\
\text { con nomenclatura incompleta (8) }\end{array}$ & $\begin{array}{c}\text { Revisa y describe la glotis, cuerdas vocales, } \\
\text { comisura, y espacio subglótico y pasa el } \\
\text { broncoscopio (10) }\end{array}$ \\
\hline
\end{tabular}

Escala $=$ 50-60 puntos competente, 40-49 parcialmente competente, 30-39 no competente. 
Tabla 2: Desempeño global de los residentes.

\begin{tabular}{|c|c|c|}
\hline \multirow{2}{*}{$\begin{array}{c}\text { Alumnos de segundo } \\
\text { y cuarto año }\end{array}$} & 1 & 2 \\
\cline { 2 - 3 } & 58 & 60 \\
\hline 1 & 58 & 56 \\
\hline 2 & 60 & 60 \\
\hline 3 & 60 & 58 \\
\hline 4 & 60 & 60 \\
\hline 5 & 60 & 60 \\
\hline 6 & 60 & 60 \\
\hline 7 & 60 & 60 \\
\hline 8 & & \multicolumn{2}{|c|}{ Evaluador } \\
\hline
\end{tabular}

cuatro primeros corresponden a los alumnos de segundo año y los cuatro últimos a los de cuarto (Tabla 2).

En el punto de evaluación de los residentes de segundo año, a pesar de que todos estuvieron en el rango de competentes, tres de ellos fueron calificados por uno o ambos evaluadores con 56 y 58 puntos.

En el residente 1, el evaluador 1 lo calificó con 58 puntos, destacando que la parte deficiente fue en cuanto a la anatomía traqueobronquial. El residente 2 fue calificado con 58 puntos por el evaluador 1 con deficiencia en cuanto a la anatomía traqueobronquial, es decir, en el conocimiento declarativo y procedimental. El evaluador 2 le dio un puntaje de 56 en cuanto a falta de medidas de protección universal, que es un conocimiento básico de cualquier médico y la preparación incompleta del paciente, que también es un conocimiento general. El residente 3 fue calificado con puntaje adecuado por ambos evaluadores con un grado de competente. El residente 4 fue calificado por el evaluador 2 con un puntaje de 58 con deficiencia en el uso de anestésico durante el procedimiento (Figura 1).

La Figura 2 muestra el desempeño de los residentes por pregunta por el evaluador 1; y en este caso, el residente 1 y 2 estuvieron de manera no satisfactoria en los rubros de anatomía de la vía aérea. En la Figura 3 vemos como el evaluador 2 calificó de manera no satisfactoria con 8 puntos al segundo residente en los rubros de medidas personales y preparación del paciente, y al residente 4 en cuanto a la aplicación de medicamento previo a la broncoscopia. En el caso de los residentes de cuarto año todos tuvieron el máximo puntaje en el manejo de la broncoscopia con los pacientes (Figura 4), tanto por calificación del evaluador 1 como del evaluador 2.

Todos cubrieron en su totalidad los conocimientos declarativos y procedimentales de manera adecuada en la realización de broncoscopias. Pero destacando mejores resultados los de tercer año en comparación con los de primer año.

\section{DISCUSIÓN}

La evaluación de la práctica clínica es una gran preocupación por parte de los docentes para tratar de no caer en apreciaciones sin fundamento y sobrevalorar o mal calificar a un alumno. En la evaluación existen instrumentos que nos permiten tener una visión clara de las cosas, dar un puntaje específico a las actividades y poder ser aplicado por varios docentes y tener resultados similares. Este es el caso de la rúbrica que usamos en este estudio.

Estos instrumentos no sólo se han aplicado en el ámbito de la ciencia, sino en la evaluación de procesos y productos de arte, diseño y actividades profesionales. En nuestro caso se aplicó en la realización de la broncoscopia para la evaluación de la práctica clínica. ${ }^{2}$

Estos instrumentos permiten, además, una evaluación auténtica. Facilitan el proceso de evaluar competencias complejas, están basados en criterios y desempeños objetivos y coherentes, describen lo que se está aprendiendo. Todos estos puntos deben ser tomados en cuenta cuando evaluamos competencias profesionales. En nuestro caso, competencias broncoscópicas que serán usadas en un futuro en la vida profesional del residente. ${ }^{5}$

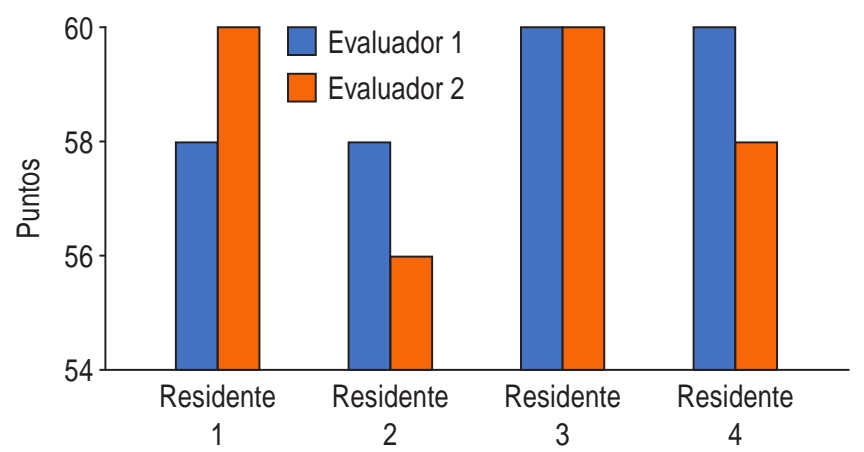

Figura 1: Desempeño de residentes de segundo año.

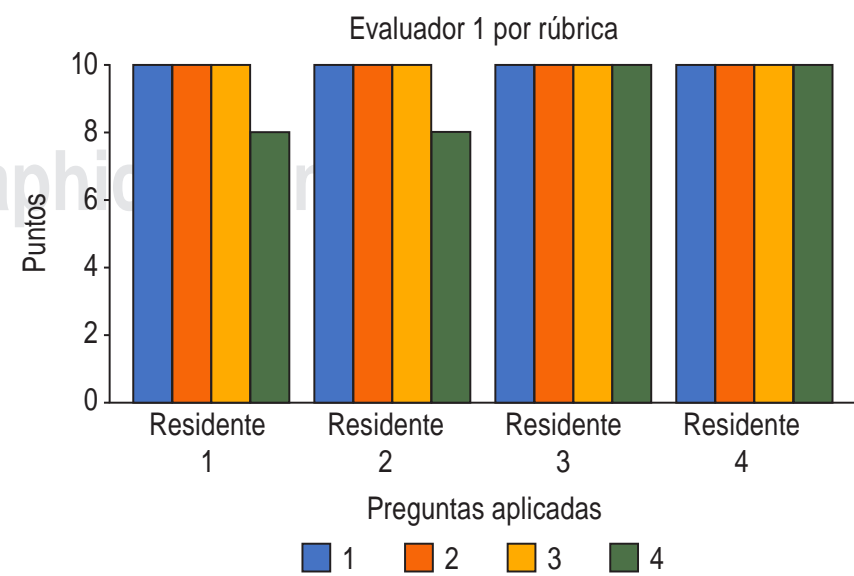

Figura 2: Evaluador 1 en residentes de segundo año. 


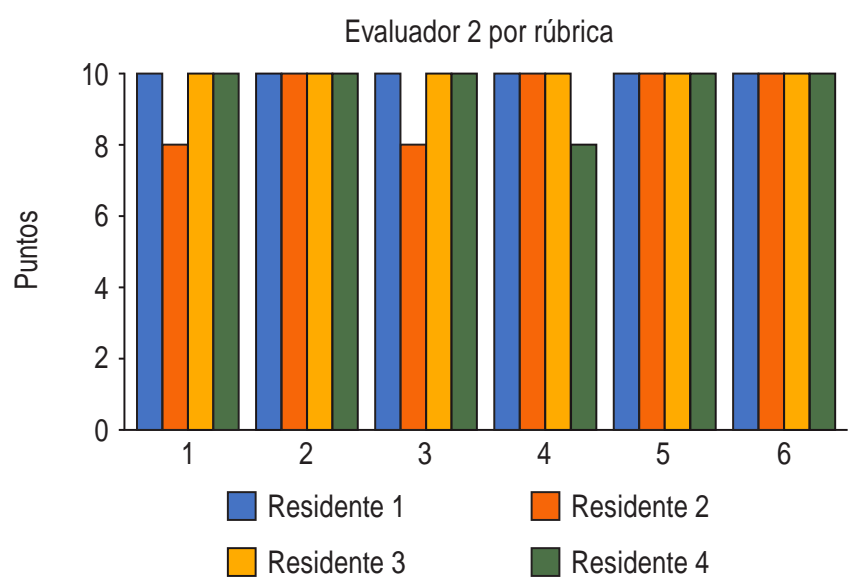

Figura 3: Evaluador 2 en residentes de segundo año.

Es importante destacar que la rúbrica permite un proceso de evaluación más valido y confiable, su valor real reside en poder ver el avance del proceso de enseñanzaaprendizaje. Resulta prioritario destacar que para la elaboración de una rúbrica se aprovecha el grado de experiencia de los profesores y se valora el grado de avance de los alumnos; también permite que el proceso de evaluación no sea dependiente de la personalidad y las variaciones del procesamiento del profesor. Esto lo pudimos aplicar al realizarla por dos profesores con experiencias docentes diferentes y tener resultados semejantes. ${ }^{6}$

Algunos autores también han usado las rúbricas para hacer expectativas de aprendizaje o suposiciones sobre las tareas a desarrollar de los alumnos, esto con la gran ventaja de poder hacer planificaciones de acuerdo con estos resultados. En nuestro caso, el resultado optimó lo que esperábamos en los residentes de cuarto año, y así fue; en cambio los de tercer año todavía tienen áreas de oportunidad para corregir. ${ }^{7}$

En medicina se han utilizado las rúbricas en muchos ámbitos de la práctica educativa debido a que son instrumentos que nos permiten valorar los aprendizajes y los productos realizados. En la enseñanza médica ha permitido al profesor especificar qué espera del alumno y cuáles son los criterios con los que va a calificar un objetivo previamente establecido. En este sentido, la realización de broncoscopia puede ser calificada o evaluada de manera satisfactoria con una rúbrica que nos permita conocer los resultados del aprendizaje de acuerdo con los objetivos de enseñanza del residente y ver de manera directa los resultados al realizar de manera correcta una broncoscopia. Los objetivos en nuestro caso son especialistas capaces de hacer procedimientos útiles y seguros para los pacientes. ${ }^{8}$

La rúbrica es considerada como una parte fundamental de la evaluación auténtica. Es una forma mediante la cual el docente puede evaluar al alumno en situaciones del «mundo real» y con ello ver la forma en que se aplican sus conocimientos y habilidades ante determinadas circunstancias. En la medicina, esta forma de evaluar es de muchísima utilidad en la práctica clínica para ver las competencias que el alumno tiene, desarrolla o refuerza. Puede ser que existan otros métodos que nos permitan evaluar estas situaciones, pero la rúbrica es un excelente método. Para nosotros fue el mejor instrumento en la evaluación del residente ante un paciente real. ${ }^{9}$

La aplicación de esta rúbrica nos permitió corroborar, a partir de la observación de dos profesores, la evolución en la adquisición de competencias, observar cómo el residente de mayor jerarquía realiza las actividades con mayor competencia que los residentes de años inferiores. ${ }^{10}$

Por tanto, la rúbrica es para nosotros el instrumento que permite corroborar lo que ya en otro trabajo previo dijimos acerca de la utilidad de la simulación como herramienta de enseñanza. Es un instrumento de evaluación in situ y de la realidad de la realización de procedimientos. Además, es un instrumento útil en la evaluación de un posgrado de medicina, como es la cirugía de tórax, en donde no hay instrumentos que puedan dar claramente resultados objetivos. ${ }^{11,12}$

Es importante destacar que los resultados obtenidos en este estudio aparte de corroborar la utilidad de la rúbrica permiten ver los avances académicos de cada uno de los médicos residentes, conocer su desempeño y sus deficiencias. En resumen, conocer su aprendizaje y ver si el programa académico cumple o no con su objetivo terminal y a la par, en parte, para evaluar el currículo del alumno y de la escuela. 13,14

Finalmente, la evaluación de un alumno de manera integral cumple con observar conocimientos, habilidades de pensamiento, habilidades y destrezas psicomotoras, actitudes y aptitudes; todas ellas, el residente las pone de manifiesto al hacer algún procedimiento, como en este caso la broncoscopia. Por ello, el evaluar in situ es útil porque se valoran de manera completa todos estos aspectos. Los

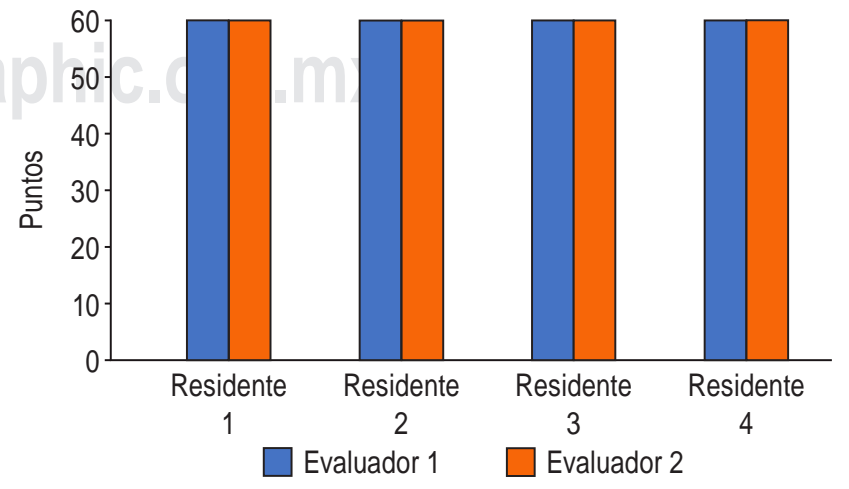

Figura 4: Evaluación de residentes de cuarto año. 
aspectos anteriores son los que recomienda el Consejo Mexicano para la acreditación de la Educación Médica. ${ }^{15}$

\section{CONCLUSIONES}

La rúbrica es un instrumento que permite evaluar los siguientes aspectos: conocimientos, habilidades de pensamiento, habilidades y destrezas psicomotoras, actitudes y aptitudes de manera reproducible y confiable en las residencias médicas.

\section{REFERENCIAS}

1. Durante MI, Martínez GA, Morales LS, Lozano SJR, Sánchez MM. Educación por competencias: de estudiante a médico. Rev Fac Med (Méx) 2011;54(6):42-50.

2. Vázquez RJA. Competencias del cirujano. Cir Gral 2012;34(Supl.1):s33-s35.

3. Díaz-Barriga AF. Enseñanza situada. Vínculo entre la escuela y la vida. México: McGraw-Hill/Interamericana; 2006.p.134-135.

4. Hernández RP. ¿Por qué es importante establecer una rúbrica de evaluación? El caso del curso de Clínica de exodoncia y cirugía. Rev Edu 2012;36(1):1-18.

5. Díaz-Barriga AF, Hernández RG. Estrategias docentes para un aprendizaje significativo. Un enfoque estructurista. México: Mc Graw-Hill/ Interamericana; 2010.p. 200-205.
6. Wolf $\mathrm{K}$, Stevens $\mathrm{E}$. The role of rubrics in advancing and assessing students learning. J Effect Taching 2007;7:3-14.

7. Andrade H, Du Y. Student perspectives on rubric-referenced assessement. Practical Asses Reseach Evaluation 2005;10(3):1-11.

8. Gómez LV, Rosales GS, Robles CA. La rúbrica como estrategia de aprendizaje en metodología de investigación en pregrado de medicina. Inv Ed Med 2019;8:30-35.

9. Galiano GJ, De Castro PM. Rúbricas para evaluación en ciencias de la salud. España: Bubok Publishing; 2010.p. 11-19.

10. Vázquez MJ, Gúzman AE, Iñiguez GM. Utilidad de la simulación de realidad virtual en la residencia de cirugía torácica en México. Neumol Cir Torax 2018;77(1):10-13.

11. Garriga SE. La rúbrica de evaluación en el posgrado. Rev Cub Inf Med 2016;8(1):144-151.

12. Marín V, Cabero AJ, Barroso OJ. La rúbrica de evaluación en el proceso de formación del docente universitario. La propuesta del proyecto DIPRO 2.0. Educar 2012;48(2):347-364.

13. Flores HF, Contreras MN, Martínez GA. Evaluación del aprendizaje en la educación médica. Rev Fac Med 2012;55(3):42-48.

14. Tyler R. Principios básicos del currículo. Buenos Aires: Troquel; 1977.

15. Consejo Mexicano para la Acreditación de la Educación Médica. Instrumento de evaluación. México: COMAEM; 2008.

Conflicto de intereses: Los autores declaran no tener conflicto de intereses. 